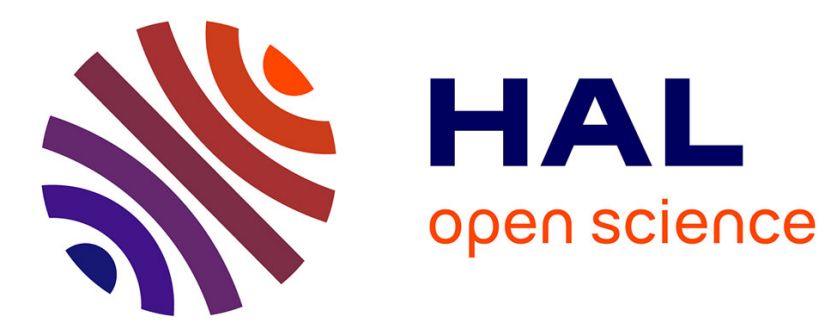

\title{
Competition between Two High-Density Assemblies of Poly(phenyl)thiols on Au(111)
}

Leonardo F. Peiretti, Paola Quaino, Frederik Tielens

\section{To cite this version:}

Leonardo F. Peiretti, Paola Quaino, Frederik Tielens. Competition between Two High-Density Assemblies of Poly(phenyl)thiols on Au(111). Journal of Physical Chemistry C, 2016, 120 (44), pp.25462 - 25472. 10.1021/acs.jpcc.6b08977 . hal-01396670

\section{HAL Id: hal-01396670 https://hal.sorbonne-universite.fr/hal-01396670}

Submitted on 14 Nov 2016

HAL is a multi-disciplinary open access archive for the deposit and dissemination of scientific research documents, whether they are published or not. The documents may come from teaching and research institutions in France or abroad, or from public or private research centers.
L'archive ouverte pluridisciplinaire HAL, est destinée au dépôt et à la diffusion de documents scientifiques de niveau recherche, publiés ou non, émanant des établissements d'enseignement et de recherche français ou étrangers, des laboratoires publics ou privés. 


\title{
The Competition between Two High Density
}

\section{Assemblies of Poly Phenyl Thiols on Au(111)}

\author{
Leonardo F. Peiretti ${ }^{\dagger}$, Paola Quaino ${ }^{* \dagger}$, Frederik Tielens $* *$ \\ † Preline, Facultad de Ingeniería Química, Universidad Nacional del Litoral, 3000, Santa Fe, Argentina \\ $\$$ Sorbonne Universités, UPMC Univ Paris 06, CNRS, Collège de France, Laboratoire de Chimie de la \\ Matière Condensée de Paris, 4 place Jussieu, 75252 Paris Cedex 05, France. \\ *,† pquaino@ fiq.unl.edu.ar \\ **.\$frederik.tielens@upmc.fr
}




\begin{abstract}
The interaction between aromatic thiols and the $\mathrm{Au}(111)$ surface is investigated using periodic DFT. Different SAM organizations were investigated, namely with different surface reconstructions (flat unreconstructed $\mathrm{Au}(111)$ and adatom $\mathrm{Au}(111)$ surface). DFT energetics on geometrically optimized SAMs and electronic analysis by means of projected density of states (PDOS) and the Crystal Orbital Hamilton Population (COHP) method, was used to interpret the adsorption property differences. It was found that the adsorption energy difference between the arylthiol chains adsorbed in T-shaped SAMs and adsorbed on flat unreconstructed Au(111) diminishes from long aryl tiols (tpt) to the short ones (dpt and mpt), indicating that the unreconstructed surface becomes more competitive energetically for short chain arylthiols (dpt and mpt). Moreover, the nature of the Au-S bond was found to be independent of the chain size. This leads us to conclude that the reconstruction, if any, is only dependent on the side chain interactions.
\end{abstract}

Keywords: Thiols, SAM, Gold, DFT, Nano 


\section{Introduction}

The adsorption of thiols on gold surfaces is known to form well-defined Self Assembled Monolayers (SAM) with a minimum of defaults. The self-assembly phenomenon has been described in detail in several reviews, ${ }^{1-2}$ and was first explained by Strong and Whitesides ${ }^{3}$. Particular attention has been paid to alkylthiols, from the smallest one, methylthiol ${ }^{4-7}$ used as a case study in physics, to long chain alkylthiols studied in applications such as coating, electronics and biological applications. ${ }^{8-9}$

The characterization of the thiol adsorption on Au surfaces has still some open questions. The adsorption site(s), the surface coverage, the surface unit cell of the SAM are starting to be well understood. Moreover, recently, we found that a surface reconstruction occurs dependently from the alkyl chain length or the type of side chain. ${ }^{10}$ Whereas the formation mechanism of the assembly on the surface is not resolved yet, ${ }^{11}$ we expect that the formation of SAMs would be also dependent of the chain lengths.

Other types of thiols have been studied in less extent such as cysteine, ${ }^{12-14}$ methionine, ${ }^{15}$ butanethiols, ${ }^{16}$ aromatic thiols ${ }^{17-18}$, etc.; some of them are summarized in the work of Costa, et al. 19. The arylthiols are used as organic molecules as surface complexing agents, so-called ligands, on gold nanoparticles allowing to introduce and managing functionality in application fields such as sensors ${ }^{1}$ or biomedicine. ${ }^{20-22}$ Such capping agents can also be adequately chosen and introduced during colloidal synthesis to control the growth of nanoparticles, and then to tune their size, shape and dispersion state. ${ }^{23-25}$ Of course, thiols form SAMs with different stabilities on the other coinage metals, $\mathrm{Cu}$ and $\mathrm{Ag}{ }^{26-27}$

Mixing of different thiols in the SAM has also been studied experimental and theoretically, and are used in specific applications such as sensor building and nanoparticles synthesis. ${ }^{23-25,28-29}$

The monolayer assembly pattern formed by aromatic thiols is less well defined compared with the one formed by alkylthiols. Unit cells comparable with the alkylthiol one, i.e., a $(2 \sqrt{3} \times 2 \sqrt{3}$ ), but also unit cells containing adatoms are described. This particular situation stimulated us to study the competition between both unit cells for monophenylthiol, diphenylthiol and triphenylthiol. In this context, we investigate the stability of the possible assemblies formed, as 
well as the chemisorption (nature of the S-Au bond) using projected density of states (PDOS) and the Crystal Orbital Hamilton Population (COHP) method.

\section{Methods}

\subsection{First Principles Calculations}

Periodic density functional theory (DFT) calculations were performed with the VASP code. ${ }^{30-33}$ The core electrons were kept frozen and replaced by pseudopotentials generated by the plane augmented wave method (PAW). ${ }^{34-37}$ The outermost shell electrons (H: $1 \mathrm{~s}^{2}, \mathrm{C}: 2 \mathrm{~s}^{2} 2 \mathrm{p}^{2}, \mathrm{~S}: 3 \mathrm{~s}^{2} 3 \mathrm{p}^{4}$, $\mathrm{Au}: 5 \mathrm{~d}^{10} 6 \mathrm{~s}^{1}$ ) were treated by means of a plane-wave basis set with a cut-off of $400 \mathrm{eV}$. The generalized gradient approximation (GGA) in the version of Perdew-Burke-Ernzerhof (PBE) was used as a functional with an accuracy on the overall convergence tested elsewhere. ${ }^{38-42}$ The application of this functional on bulk Au resulted in a theoretical lattice parameter of $\mathrm{a}_{0}{ }^{\mathrm{Au}}=4.18$ $\AA$. Within the typical margin of error, the constant agree with the experimental data $\left(\mathrm{a}_{0}=4.08 \AA\right)$ reported in the literature. ${ }^{43}$ The parameterization of the k-points sampling of the Brillouin zone based on the Monkhorst-Pack grid $^{44-45}$ was considered. The parameter were increased systematically until the change in the absolute energy was less than $10 \mathrm{meV}$. A grid of $(5 \times 7 \times 1)$ and $(5 \times 5 \times 1)$ k-points were used for $(2 \sqrt{3} \times \sqrt{3})$ and $(2 \sqrt{3} \times 2 \sqrt{3})$ unit cells, respectively. Spin polarizations were considered for the isolated radicals, whereas for the adsorbed systems no spin polarization was found. In order to take into account van der Waals interactions in the SAM (Self-Assembled Monolayer) system, the DFT-D3 ${ }^{46}$ approach of Grimme ${ }^{47}$ was used, which consists in adding a semi-empirical dispersion potential to the conventional Kohn-Sham DFT energy.

\subsection{Modeling}

To study the thiol adsorption process two surfaces were considered: a flat $\mathrm{Au}(111)$ and a nanostructured surface represented by two gold adatoms adsorbed on $\mathrm{Au}(111)$, identified as $\mathrm{Au}_{\mathrm{ad}^{-}}$ $\mathrm{Au}(111)$. The former surface was modelled using a $(2 \sqrt{3} \times \sqrt{3})$ supercell with 4 metal layers. For the nanostructured system, a $(2 \sqrt{3} \times 2 \sqrt{3})$ supercell with also 4 metal layers plus two gold 
adatoms was used. For specific details, see Figure 1. The behavior of three different poly-phenylthiols were considered: benzenethiol (mpt or monophenylthiol); 1,1'-biphenyl-4-thiol (dpt or diphenylthiol) and 1,1',4',1"'-terphenyl-4-thiol (tpt or triphenylthiol), and they were compared with the 1-dodecanethiol (C12) which has been taken as reference system. Figure 2 shows the investigated thiols in their radical forms in gas phase.
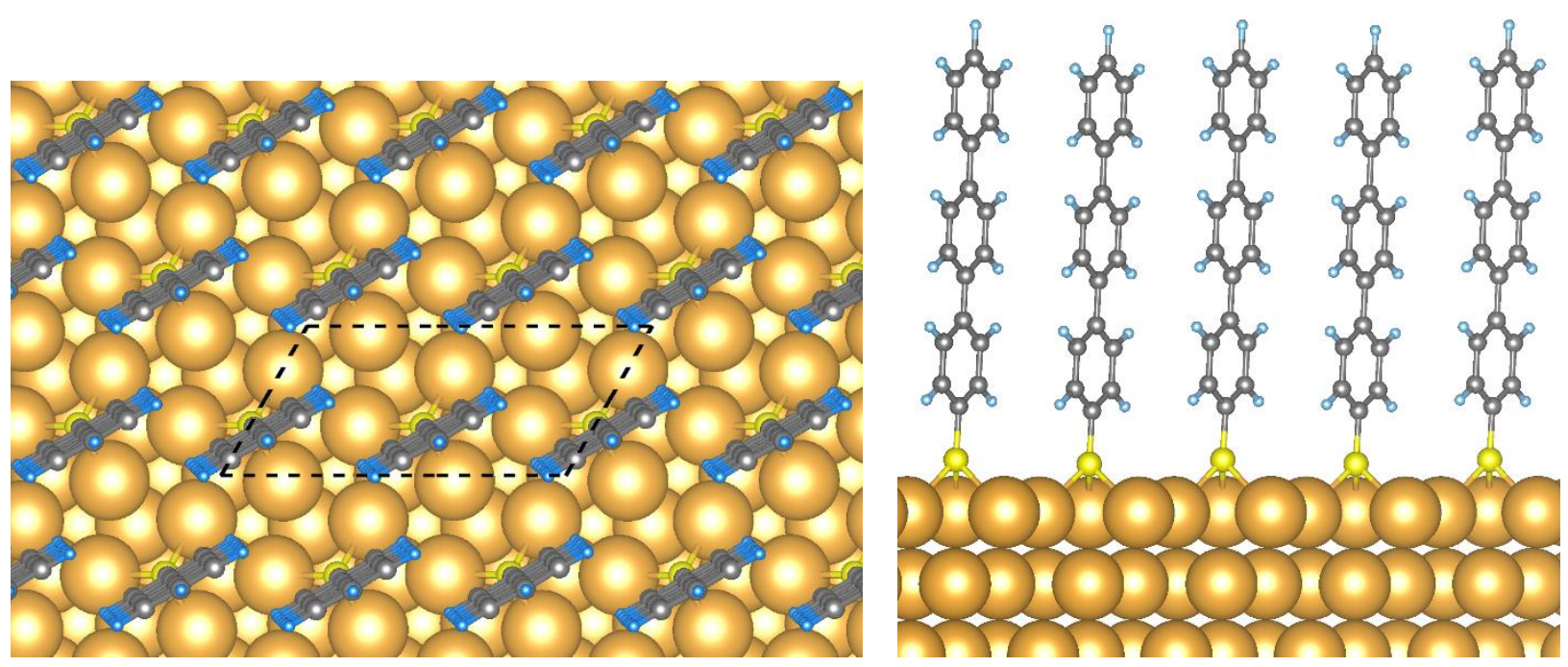

(a)
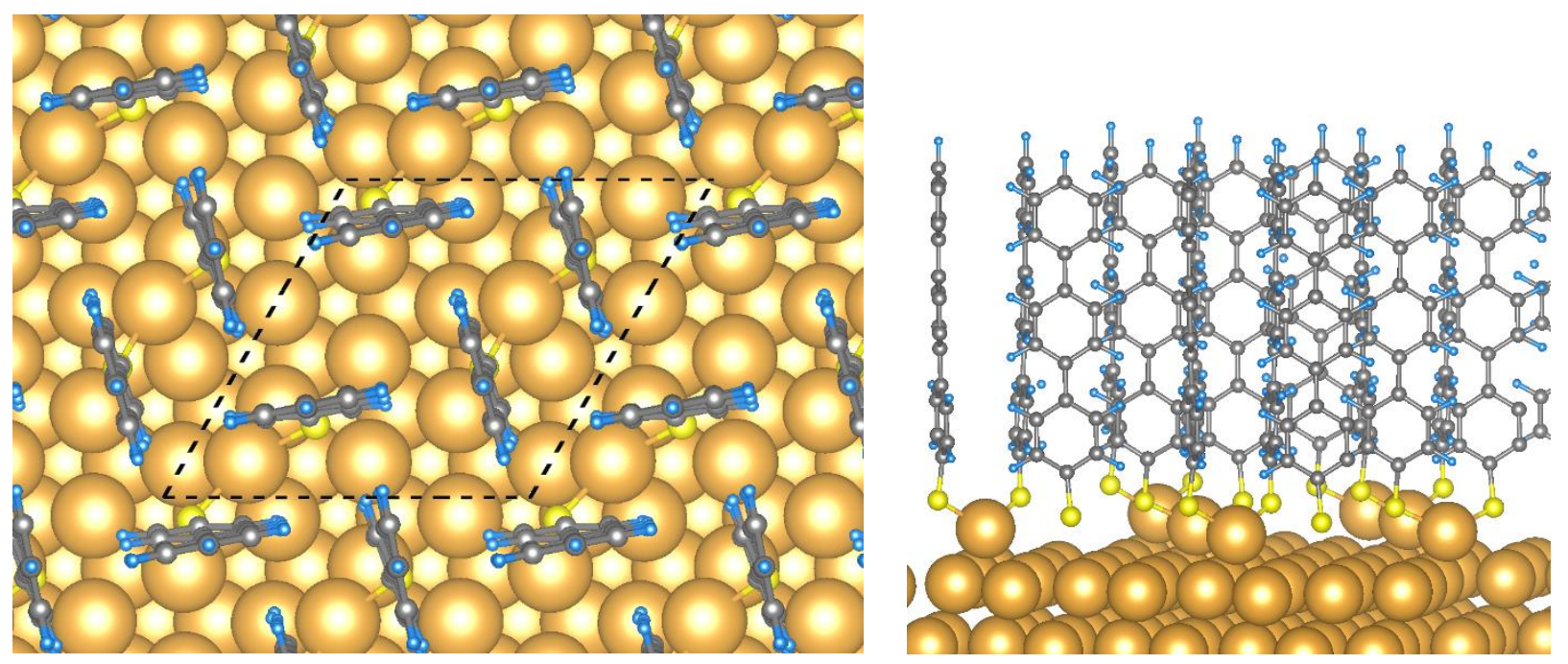

(b)

Figure 1. Au(111) surfaces used for the thiols adsorption study. (a) Flat surface, (b) nanostructured surface. 


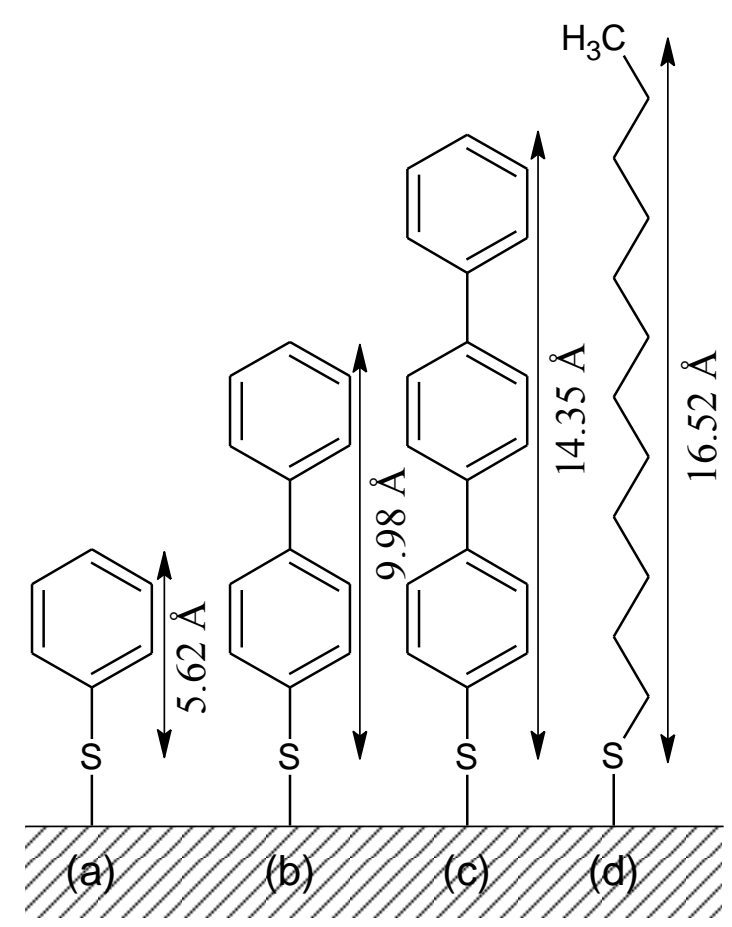

Figure 2. Thiols analyzed: (a) mpt, (b) dpt, (c) tpt and (d) C12.

In all the calculations a vacuum corresponding to $20 \AA$ was used. For all the systems, the two bottom layers were fixed at the next-neighbor distance corresponding to bulk and all the other layers plus the thiols were allowed to fully relax. The relaxations were performed within conjugate-gradient minimization scheme, and the convergence criterion was achieved if the total forces were less than $0.02 \mathrm{eV} / \AA$.

The adsorption was investigated in all the high symmetry sites for the pure flat surface, and the most favorable one is reported in the present contribution. To study the self-assembled monolayer (SAM), two adsorbed thiols were considered on the flat surface. In the case of the nanostructured one, the thiols were adsorbed on a gold adatom in pairs, with a pattern where the phenyl chains were parallel and perpendicular respect to each other as shown in Figure 1(b). We opted for the T-shaped adatom configuration, according to previous STM observations on flat Au(111) surfaces. $^{48}$

\subsection{Theoretical description}

The adsorption energies $\Delta E_{\text {ads.disp }}$ per chain of thiol on the gold surface has been calculated as follows: 


$$
\Delta E_{\text {ads.disp }}=\Delta E_{\text {ads.PBE }}+\Delta E_{\text {disp }}
$$

where $\Delta E_{\text {ads.PBE }}$ is the adsorption energy calculated from the pure DFT-PBE electron energies. $\Delta E_{\text {ads.PBE }}$ was calculated according to the following equation:

$$
\Delta E_{\text {ads.PBE }}=\frac{1}{n}\left(E_{\text {thio//Au(111) }}-n E_{\text {thiol }}-E_{\mathrm{Au}(111)}\right)
$$

where $E_{\mathrm{thiol} / \mathrm{Au}(111)}, E_{\mathrm{thiol}}$, and $E_{\mathrm{Au}(111)}$ are the total electronic energies of the adsorption complex formed by thiol, the isolated thiol under its radical form, and the $\mathrm{Au}(111)$ slab, obtained after separate geometry optimization, respectively. The variable $n$ represents the number of thiols per unit cell.

As it was mentioned previously, the dispersion interaction energy ( $\left.\Delta E_{\text {disp }}\right)$ was evaluated by means of the DFT-D3 approach. ${ }^{49}$ Then, the adsorption energy of one thiol chain in a perfectly

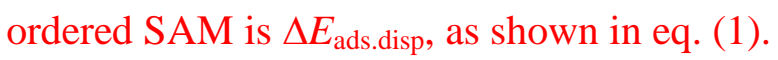

The Au-S binding energy ( $\Delta E_{\text {bind }}$ ) has been evaluated, with or without dispersion forces, considering the adsorption of thiolates at low coverage, i.e., with the adsorbate-adsorbate interactions negligible:

$$
\Delta E_{\text {bind }}=\frac{1}{n}\left(E_{\text {thiol/Au(111) }}-E_{\text {thiol.SAM }}-E_{\mathrm{Au}(111)}\right)
$$

with $E_{\text {thiol.SAM }}$ being the single point (not geometrically relaxed) electronic energy of the thiol radicals ( 2 in the $2 \sqrt{3} \times \sqrt{3}$ unit cell and 4 in the $2 \sqrt{3} \times 2 \sqrt{3}$ supercell) in the configuration of the SAM but without considering the $\mathrm{Au}(111)$ slab.

In this context, an approximation for the interchain interaction energy ( $\left.\Delta E_{\text {int.chain }}\right)$, with or without dispersion forces, can be obtained as following:

$$
\Delta E_{\text {int.chain }}=\Delta E_{\text {ads }}-\Delta E_{\text {bind }}
$$

At the pure PBE level for arylthiols, the intermolecular interactions were calculated and they resulted to be repulsive (Table 1 and Table 2), due to the omission of the stabilizing dispersion intermolecular interactions in the assembly at this calculation level. In order to correct this point, calculations at a more advanced level were performed by considering dispersion forces, which 
are of prime importance when interactions between aromatic groups or alkyl chains are at play. Although this refinement lowered the Au-S binding energy, the trend observed was similar than that obtained at the PBE level. On the other hand, the contribution of the intermolecular interactions was dramatically modified and became attractive, as expected. This result validates our procedure for taking into account dispersion interactions. ${ }^{19}$

Eventually one could also breakdown the adsorption energy separating the molecule and surface deformation energies, which are implicitly included in the different energies. However, in order to keep the discussion concise they were not discussed, since they do not influence the discussion on the relative stability between the arrangements studied here.

\section{Results and discussion}

\subsection{Energetics of the phenylthiols on gold surfaces}

The alkylthiol adsorption on metal surfaces is a well-reported topic, theoretical and experimentally. ${ }^{10,50-54}$ Nevertheless, there is less information about the adsorption behavior of phenyl or phenyl/alkylthiol adsorption on metals, especially discussed in terms of dispersion interactions, and the competition between high-density and low-density assemblies. Very recently, the effect of dispersion was shown to be important in the adsorption geometry of alkylthiols. ${ }^{55-56}$

In this context, we have investigated the adsorption of three phenylthiols; named mpt, dpt and tpt as indicated in section 2.2. in two different configurations: parallel (Figure 3) and T-shaped (Figure 4). The adsorption, binding and inter-chain energies for the phenylthiols adsorbed on the flat and the nanostructured surface are shown in Table 1 and Table 2, respectively. For comparison, $\mathrm{C} 12$ thiol (1-dodecanethiol) on a $\mathrm{Au}(111)$ surface is added as well (Figure 5). 


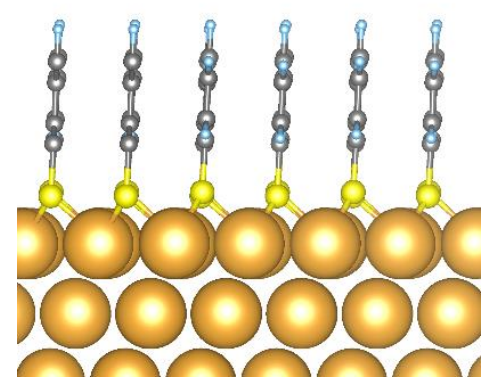

(a)

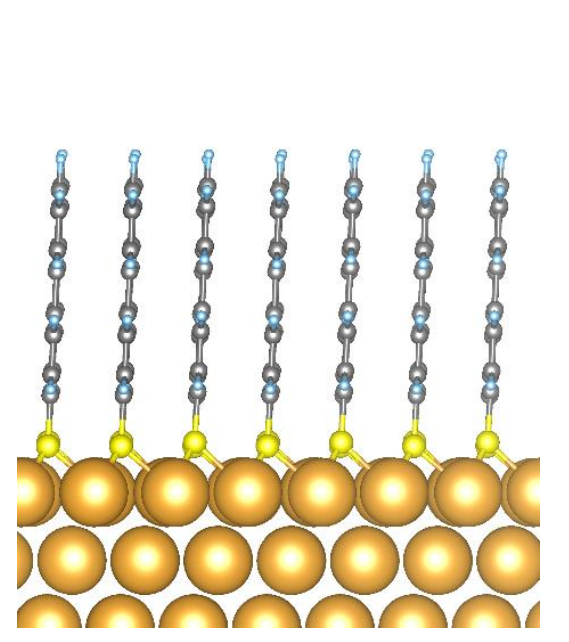

(b)

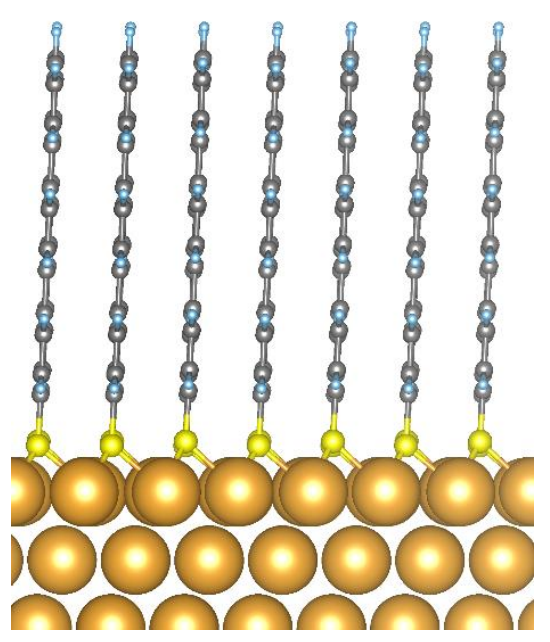

(c)

Figure 3. Optimized thiols on the $\operatorname{Au}(111)$ flat surface. (a) mpt, (b) dpt, (c) tpt.

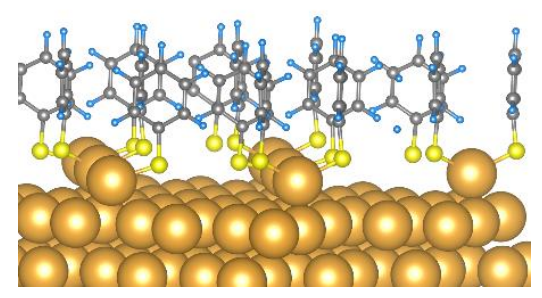

(a)

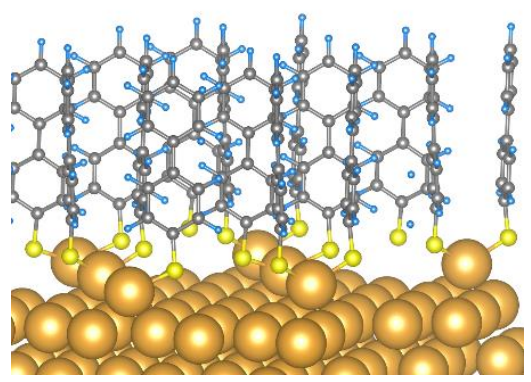

(b)

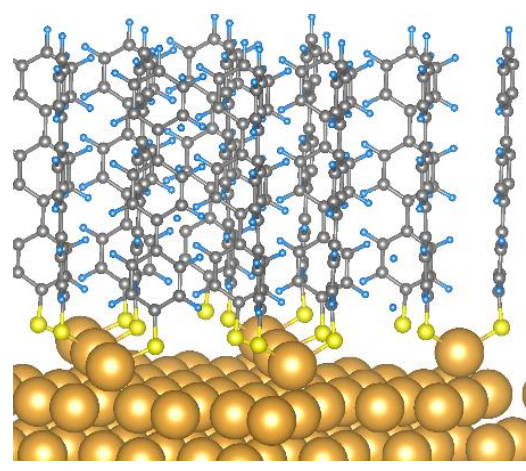

(c)

Figure 4. Optimized thiols on the $\operatorname{Au}(111)$ nanostructured surface. (a) mpt, (b) dpt, (c) tpt.

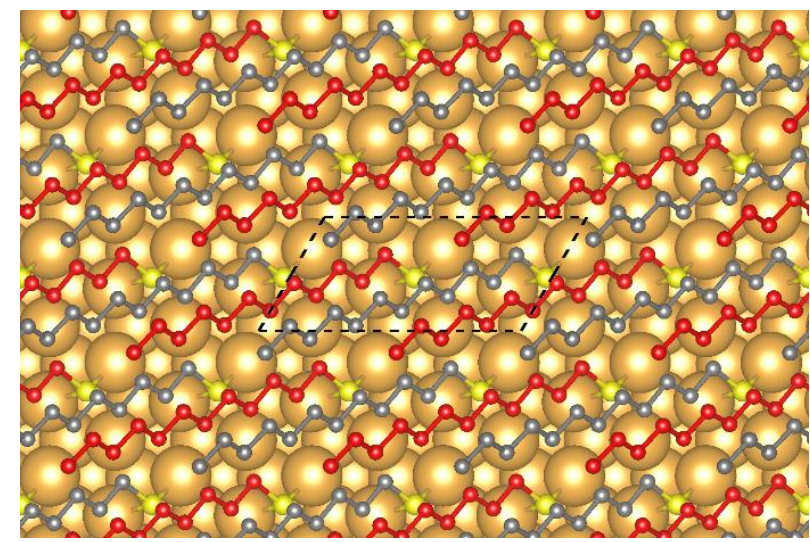

(a)

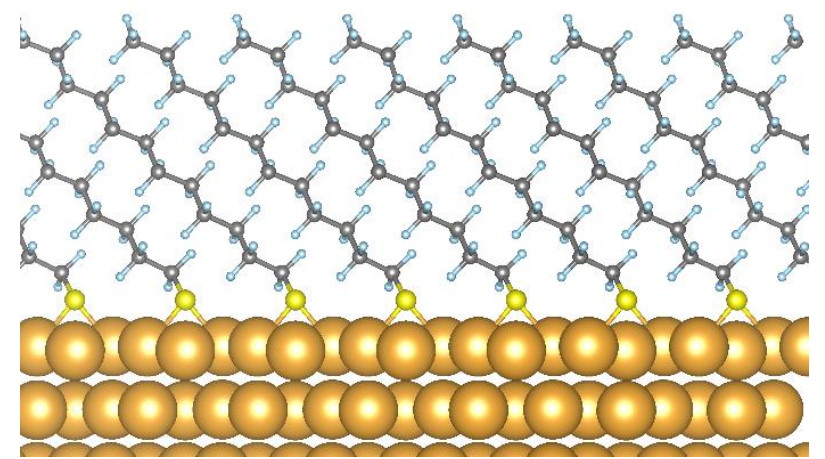

(b)

Figure 5. Optimized 1-dodecanethiol (C12) on the Au(111) flat surface. (a) top view (hydrogens were hidden and one $\mathrm{C} 12$ was colored in red for clarity), (b) side view. 
Table 1. Adsorption ( $\left.\Delta E_{\text {ads }}\right)$, binding $\left(\Delta E_{\text {bind }}\right)$ and inter-chain $\left(\Delta E_{\text {int.chain }}\right)$ energies for several thiols (mpt, dpt, tpt, and C12) on a flat gold surface. All energies are in $\mathrm{eV}$.

\begin{tabular}{ccccccccc}
\hline $\begin{array}{c}\text { Energy } \\
(\mathbf{e V})\end{array}$ & \multicolumn{2}{c}{ mpt } & \multicolumn{2}{c}{ dpt } & \multicolumn{2}{c}{ tpt } & \multicolumn{2}{c}{ C12 } \\
\cline { 2 - 9 } & PBE & PBE-D3 & PBE & PBE-D3 & PBE & PBE-D3 & PBE & PBE-D3 \\
\hline $\boldsymbol{\Delta} \boldsymbol{E}_{\text {ads }}$ & -0.92 & -2.04 & -0.70 & -2.25 & -0.58 & -2.53 & -1.53 & -3.32 \\
\hline $\boldsymbol{\Delta} \boldsymbol{E}_{\text {bind }}(\mathbf{S}-\mathbf{A u})$ & -1.34 & -2.12 & -1.26 & -2.06 & -1.26 & -2.05 & -1.78 & -2.60 \\
\hline $\boldsymbol{\Delta} \boldsymbol{E}_{\text {int.chain }}$ & 0.42 & 0.08 & 0.56 & -0.19 & 0.68 & -0.48 & 0.25 & -0.72 \\
\hline
\end{tabular}

Table 2. Adsorption $\left(\Delta E_{\text {ads }}\right)$, binding $\left(\Delta E_{\text {bind }}\right)$ and inter-chain $\left(\Delta E_{\text {int.chain }}\right)$ energies for several thiols (mpt, dpt, tpt, and C12) on a nanostructured gold surface. All energies are in eV.

\begin{tabular}{ccccccc}
\hline $\begin{array}{c}\text { Energy } \\
(\mathbf{e V})\end{array}$ & \multicolumn{2}{c}{ mpt } & \multicolumn{2}{c}{ Dpt } & \multicolumn{2}{c}{ tpt } \\
\cline { 2 - 7 } & PBE & PBE-D3 & PBE & PBE-D3 & PBE & PBE-D3 \\
\hline $\boldsymbol{\Delta} \boldsymbol{E}_{\text {ads }}$ & -1.34 & -2.27 & -1.20 & -2.50 & -1.22 & -2.92 \\
\hline $\boldsymbol{\Delta} \boldsymbol{E}_{\text {bind }}(\mathrm{S}-\mathrm{Au})$ & -1.63 & -2.20 & -1.46 & -1.99 & -1.43 & -1.96 \\
\hline $\boldsymbol{\Delta} \boldsymbol{E}_{\text {int.chain }}$ & 0.29 & -0.07 & 0.26 & -0.49 & 0.21 & -0.96 \\
\hline
\end{tabular}

An analysis of our results showed that the adsorption energy of C12 $(-3.63 \mathrm{eV})$ is larger than those for the phenylthiols (mpt, dpt, tpt) in both assemblies, parallel and T-shaped. The energy difference between the alkylthiol $(\mathrm{C} 12)$ and the arylthiols increases from tri-, di- to monophenylthiols, in both configurations when dispersion forces are taken into account. A comparison between the dodecanethiol and the triphenylthiol, taken as a reference for all the investigated thiols, indicates that stacking methylene groups in alkylthiols is more favorable than the aromatic ring stacking (Figure 3 and Figure 5). This trend is explained by the geometrical restraints of the aromatic groups respect to the (long) alkyl chains that have more atoms at the optimum distance to reach a greater stability. ${ }^{57}$ The interactions between aromatic groups, which show $\pi-\pi$ stacking have been studied in detail before, ${ }^{58}$ and have also a strong stabilizing effect. However, since the dispersive interactions are additive, in our case not even the triphenyl thiol reach the stabilization of a C12 long alkyl chain.

An interesting result is that the adsorption energy, which is also a descriptor for the SAM stability, showed that the T-shaped arrangement is in all cases more energetically favorable than 
the parallel scheme. When dispersion forces are taken into account, the attractive intermolecular interactions increase monotonously with the number of phenyl groups in the side chain, as expected from $\pi / \pi$ ( $\pi$ stacking for parallel phenyl groups) and $\mathrm{C}-\mathrm{H} / \pi$ (for close to perpendicular phenyl groups) interactions. ${ }^{49}, 59$ These systems became less favorable in the following order (Table 1 and Table 2): mpt $<$ dpt $<$ tpt. Quantitatively, adding a new phenyl ring in a monophenylthiol leads to a change in the adsorption energy of approx. $0.25 \mathrm{eV}$ in both configurations. However, the addition of a ring in a dpt molecule evidences changes of 0.28 and $0.42 \mathrm{eV}$ for the parallel and the T-shaped conformations, respectively; resulting in a significant stabilizing effect for the T-shaped SAM.

Since the stabilization is measured by the adsorption energy and the Au-S bond energy is almost constant, the difference between both adsorption energies (parallel and T-shaped) is due to the dispersion interactions. It is interesting to note that the parallel $\pi-\pi$ interaction is less stable probably due to the geometrical restraints in which the molecules are trapped within the SAM configuration, which forbids the thiols to reach an optimal intermolecular distance. On the other hand, the unit cell containing the adatoms allows a more favorable geometrical configuration than that obtained with the flat $\mathrm{Au}(111)$, which is in accordance with experimental information. ${ }^{60-61}$

According to eq. (4), the adsorption energy can be subdivided into two different energy contributions: intermolecular interactions $\left(\Delta E_{\text {int.chain }}\right)$ and the binding energy $\left(\Delta E_{\text {bind }}\right)$. Regarding to the former, Table 1 and Table 2 show that the intermolecular interactions are stronger in $\mathrm{C} 12$ than between the phenyl chains. However, it should be mentioned that for the case of the tpt on the $\mathrm{Au}_{\mathrm{ad}} / \mathrm{Au}(111)$ surface, the interchain energies are highly comparable respect to the $\mathrm{C} 12$, with an interchain energy difference of about $|0.08| \mathrm{eV}$, which is within the expected error bar in a DFT calculation $(0.1 \mathrm{eV})$. The dense packing achieved by $\mathrm{C} 12$ allows a free energy minimization with a high degree of van der Waals interactions between chains; ${ }^{62-63}$ situation that cannot be replicated by the tpt in the same flat surface. ${ }^{64}$ However, the gold nanostructuration raises the van der Waals interactions for the tpt chains, which are now closer but less packed than C12. Additionally, phenyl chain SAMs arrange in configurations that are more complex and change more easily between conformations than the akyl chain SAMs. As expected, the rigidity in the configurations for the arylthiols increases with the number of phenyl rings due to larger interactions between the chains. Interestingly, for all the investigated arylthiols on both surfaces, 
$\mathrm{Au}(111)$ and $\mathrm{Au}_{\mathrm{ad}} / \mathrm{Au}(111)$, the binding energy values for the S-Au bond are almost similar and around $2.0-2.2 \mathrm{eV}$, which indicates that the adsorption energy is mainly determined by the nature of thiol chains and the intermolecular interaction forces that exist between those chains. Hence, the changes in the Au-S bond play only a minor role in the differences in surface affinities for this kind of ligand. This effect was particularly pronounced between dpt and tpt on both configurations, see Table 1 and Table 2.

It is well accepted from experimental and theoretical viewpoints that the thiol adsorption on a $\mathrm{Au}(111)$ surface induces a reconstruction phenomenon, involving the formation of thiol- $\mathrm{Au}_{\mathrm{ad}^{-}}$ thiol moieties, ${ }^{60,65-68}$ which are even more stable than the assembly thiol- $\mathrm{Au}_{\mathrm{ad}}{ }^{61}$ Hence, a detailed examination of the adsorption energies let us predict that the parallel configuration could transform into a T-shaped structure in order to gain stability, and the easiness of this process would decrease by increasing the number of phenyl rings (Figure 6). In order to do this, the thiols should extract a gold atom from the surface and change their configuration from parallel to $\mathrm{T}$ shaped. This phenomenon is under discussion nowadays, but some progress has been done for molecules as the methylthiol/Au(111). ${ }^{60-61}$ Another possible way to generate adatoms is the diffusion of $\mathrm{Au}$ atoms on the surface originating from steps and corners.

A study of the flat surface allowed finding that the distances between the gold atoms implicated in the $\mathrm{S}-\mathrm{Au}$ bond showed an increase of $8.7 \%$ respect to the nearest neighbor distance from the bulk. However, this increase was not observed between another whatever two gold atoms, in fact, the distance decreased by $4.8 \%$. The pressure release by the extraction of the adatom could be one of the driven forces for the phase change. The presence of two different sulfur-gold interfaces -flat $(\mathrm{S}-\mathrm{Au})$ and nanostructured $\left(\mathrm{S}-\mathrm{Au}_{\mathrm{ad}}\right)$ surfaces- suggests a competition between the SAM organization and the existence of intermolecular forces. 


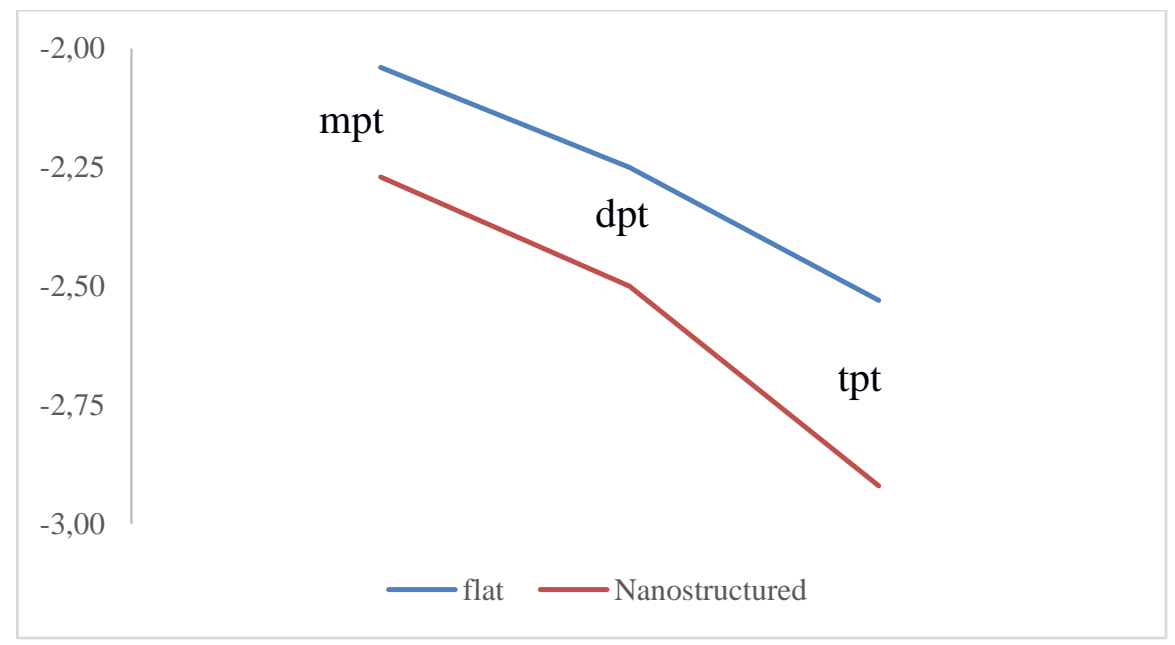

Figure 6. Adsorption energies for each thiol studied (mpt, dpt and tpt) adsorbed on both surfaces: flat and nanostructured.

\subsection{Nature of the phenylthiol-Au bond}

The interpretation of the electronic structure of these systems: thiol-Au(111) and thiol$\mathrm{Au}_{\mathrm{ad}} / \mathrm{Au}(111)$ where thiol $=\mathrm{mtp}, \mathrm{dpt}, \mathrm{tpt}$; is rather complicated due to the presence of several interactions between the adsorbed species as well as the electronic states of the metal surface. Therefore, in order to understand the nature of these interactions, the projected density of states (PDOS) was examined. The PDOS presented in this contribution were not normalized and the energy values are referred to the Fermi level taken as zero energy. In addition, and for the sake of completeness, the nature of bonding/antibonding character of these molecular orbitals was studied using the Crystal Orbital Hamilton Population (COHP) method. We will not explain the details of this well-known method but we will summarize important remarks about the information that can be obtained from COHP. For further information about this method, see the work of Dronskowski and Bloechl ${ }^{69}$. The COHP is a bonding indicator for solid systems that is constructed by generating an overlap population-weighted density of states. It is important to emphasize that negative values of COHP curves correspond to bonding states, whereas the positives are related to the antibonding states. 

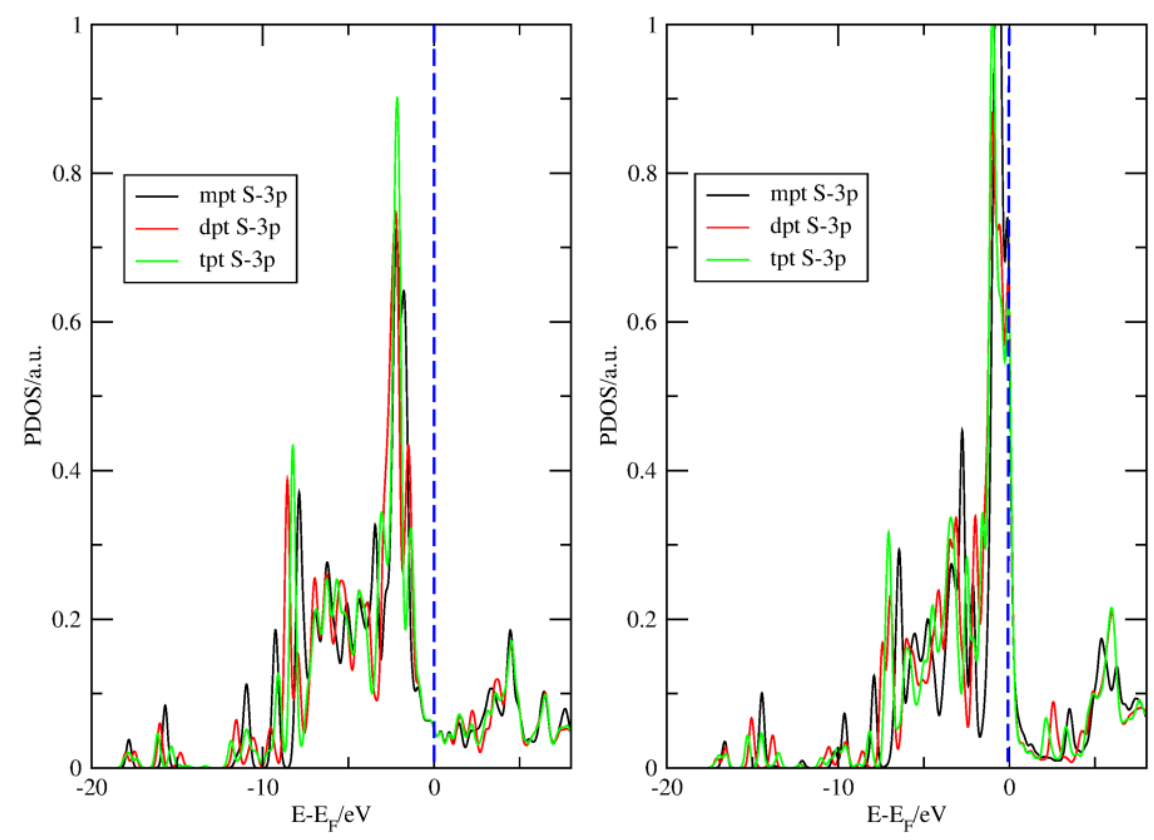

Figure 7. PDOS for the S $3 p$ states for the three phenylthiols studied, adsorbed on the flat Au(111) surface (left panel) and on the $\mathrm{Au}_{\mathrm{ad}} / \mathrm{Au}(111)$ (right panel).

Figure 7 shows a comparison between the $3 p$ states of the sulfur presented in the three investigated phenylthiols adsorbed on a flat (left panel) and on a nanostructured (right panel) $\mathrm{Au}(111)$ surface. As it can be seen, the PDOS profiles for the sulfur atom are practically the same for mpt, dpt and tpt in each gold surface. Hence, the nature of the Au-S bond seems to be independent of the chain size. These results are in agreement with the binding energy values displayed in Table 1 and Table 2, which remain almost unchanged with the chain length. For the thiol- $\mathrm{Au}_{\mathrm{ad}} / \mathrm{Au}(111)$ system, the $3 p$ states of the sulfur directly bonded to the adatom appeared at more positive energies, due to the up-shift in the $d$-and $s p$-bands of the substrate. Changes in the electronic properties of nanostructured surfaces are expected due to alterations in the geometry in comparison to flat surfaces (Figure 8). Hence, the $d$ - and $s p$-bands are shifted to more positive energy values. Furthermore, in the pure $\mathrm{Au}_{\mathrm{ad}} / \mathrm{Au}(111)$ surfaces the $d$-bands become thinner indicating stronger electron localization. These changes in the electronic properties enhance the surface reactivity of the nanostructured surface respect to the flat one. The higher adsorption energies on the nanostructured surface are the proof for this phenomenon (Table 1 and Table 2). 

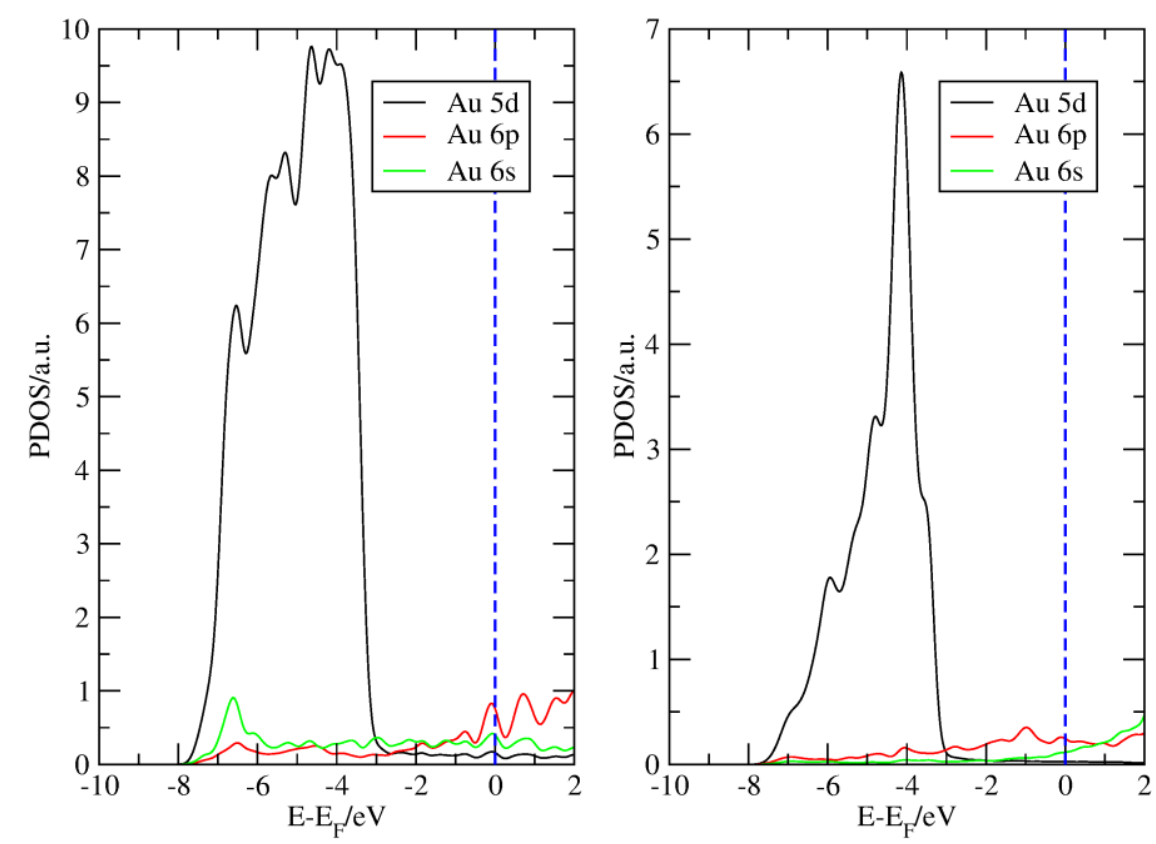

\section{Figure 8. PDOS for the pure flat $\mathrm{Au}(111)$ (left panel) and the pure $\mathrm{Au}_{\mathrm{ad}} / \mathrm{Au}(111)$ (right panel)} surfaces.

Seeking for a better understanding of the S-Au bond nature, we performed a detailed analysis of the $3 p$ states of the sulfur atom and its interaction with the $s p$ - and $d$-bands of the surface. The $\mathrm{Au}$ atom directly bonded to the $\mathrm{S}$ of the thiol was selected for the analysis in both systems. In the case of the flat surface, the Au-S bond was formed by a S bonded to three gold atoms, which exhibit the same PDOS profile. In the case of the T-shaped system, the S-Au bond between the adatom and both sulfur atoms showed similar PDOS profiles as well. Figure 9, Figure 10 and Figure 11 show the total $3 p$ states of the sulfur in the triphenylthiol with the $s^{-}, p$ - and $d$-bands of gold in both configurations as a reference. Monophenylthiol and diphenythiol are not shown, since their PDOS profiles were similar to the one of tpt. The COHP curves were also added to help in the interpretation of the bonding/antibonding contributions. Left and right columns correspond to $\mathrm{Au}(111)$ and $\mathrm{Au}_{\mathrm{ad}} / \mathrm{Au}(111)$ surfaces, respectively. 

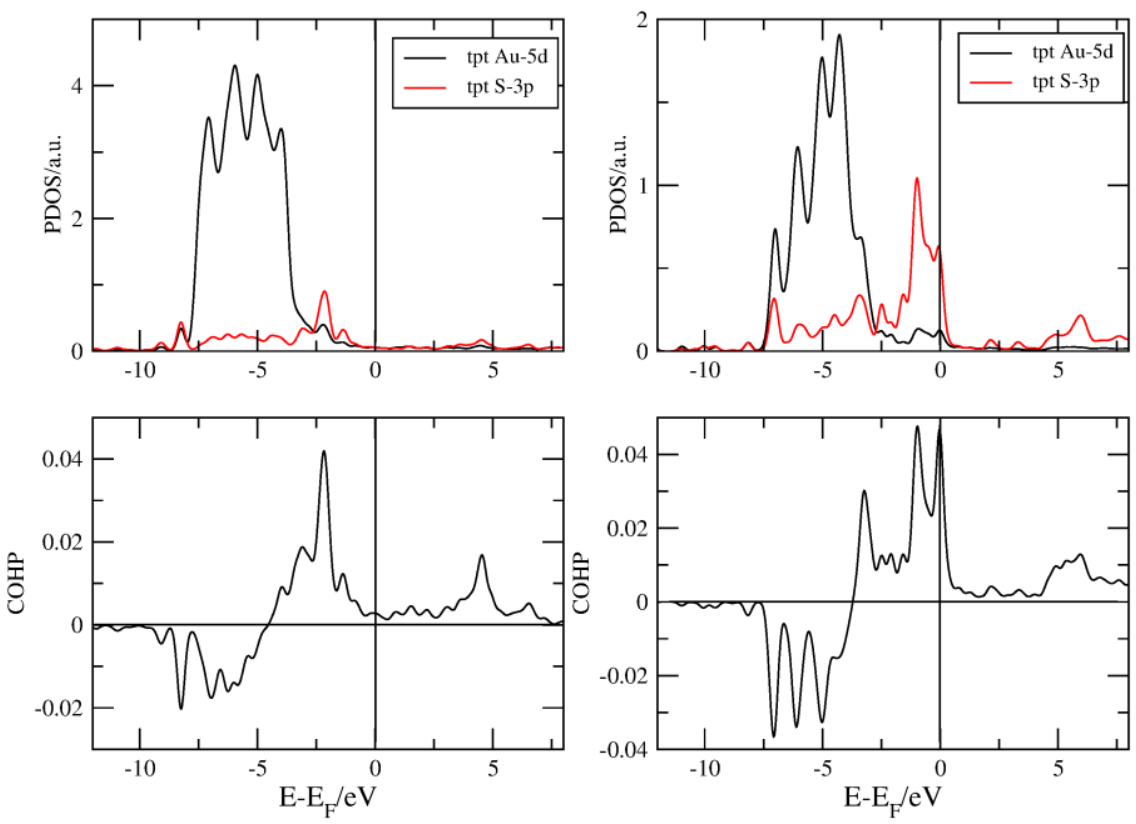

Figure 9. PDOS and COHP for the Au $5 d$ state with the S $3 p$ state of the tpt adsorbed on the flat (left column) and on the nanostructured (right column) surfaces.
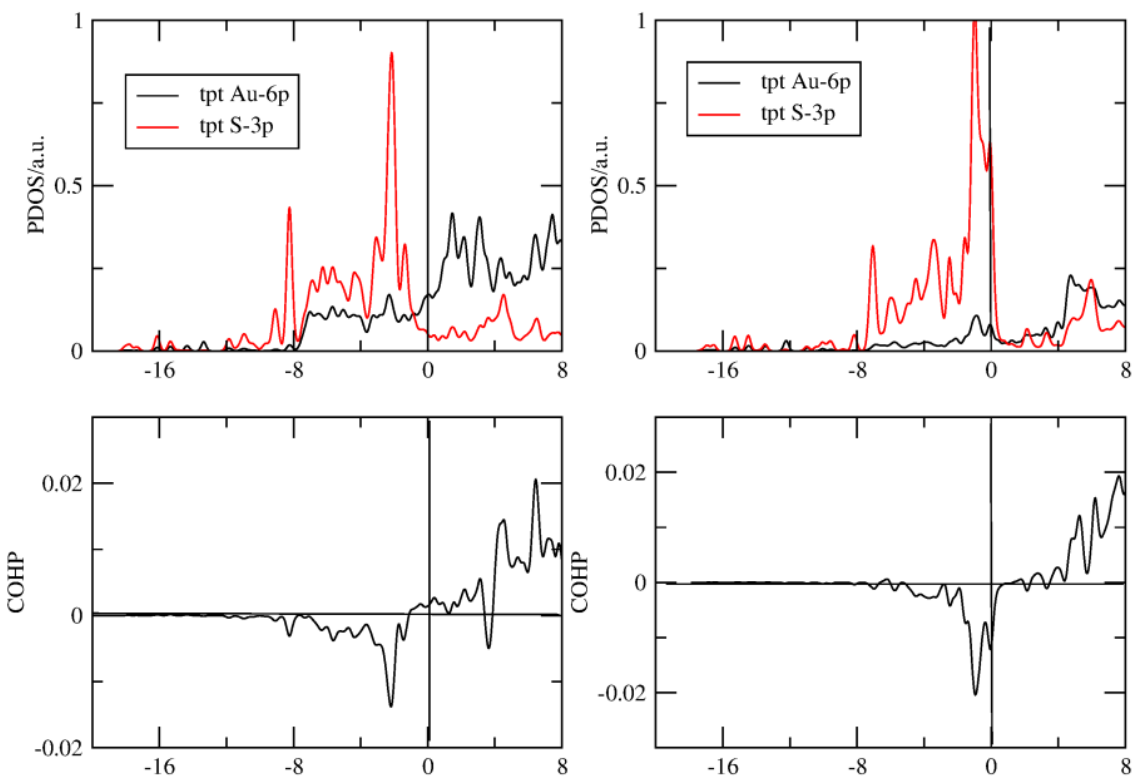

Figure 10. PDOS and COHP for the Au $6 p$ state with the S $3 p$ state of the tpt adsorbed on the flat (left column) and on the nanostructured (right column) surfaces. 

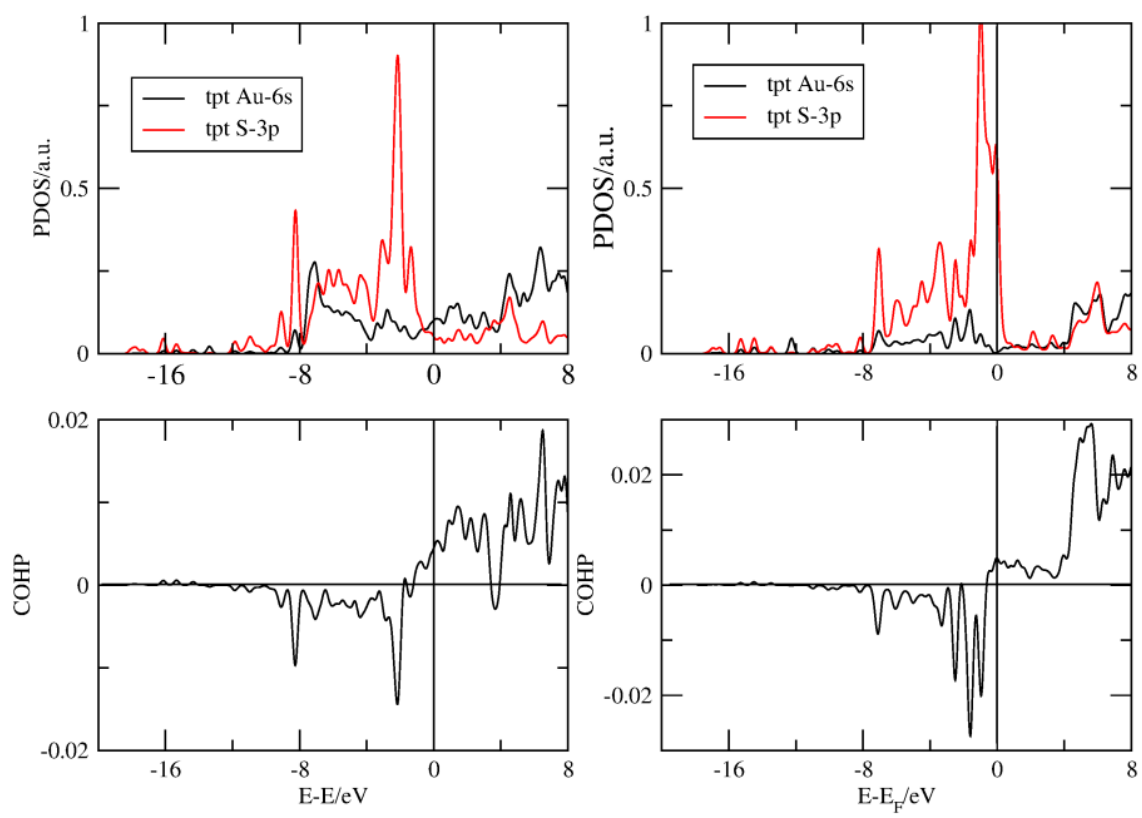

\section{Figure 11. PDOS and COHP for the Au $6 s$ state with the S $3 p$ state of the tpt adsorbed on the flat (left column) and on the nanostructured (right column) surfaces.}

Upon adsorption, the $3 p$ states of the thiol become broader and shifted to negative energies in comparison to the isolated radical (not shown) due to the interplay with the metal surface. The $d$ bands of $\mathrm{Au}(111)$ and $\mathrm{Au}_{\mathrm{ad}} / \mathrm{Au}(111)$ lie between $-8 \mathrm{eV}$ and $-3 \mathrm{eV}$ below the Fermi level, with their bonding and antibonding contributions of the $3 p$ states - caused by the interaction with the $d$ band- filled (Figure 9). Thus, the $d$-band does not contribute significantly to the adsorption bond, which is therefore dominated by the interaction with the $s p$-band. The $s$ - and $p$ - states of the surfaces are affected by the presence of the thiol, since the bonding and antibonding contributions appeared below and above the Fermi level, respectively; stabilizing the S-Au bond (Figure 10 and Figure 11). Thus, COHP plots allowed us to confirm that the $\mathrm{S}$-Au bond shows mainly a $\mathrm{S}(3 p)$ $\mathrm{Au}(6 s p)$ character, because the bonding state is located bellow the Fermi level while the antibonding state is found above the Fermi level. Obviously, this behavior indicates a strong bonding between $\mathrm{S}(3 p)-\mathrm{Au}(6 s)$ (Figure 11) and $\mathrm{S}(3 p)-\mathrm{Au}(6 p)$ (Figure 10). On the other side, the analysis of the COHP profiles for the $\mathrm{S}(3 p)-\mathrm{Au}(5 d)$ bond showed a different behavior: the bonding and antibonding states were both located bellow the Fermi level, revealing that no bond is formed. 
It should be mentioned that our analysis is mainly focused in the Au-S nature, a more complete analysis is out of the scope of this work, which should consider highly entangled interactions: the participation of the $3 s$ states of the sulfur, the metal states, and all the states of the rest of the thiol chain formed by $\mathrm{C}$ and $\mathrm{H}$.

Finally, the corresponding rearrangement of electronic charge upon the formation of the assembly of thiols can be obtained by subtracting the charge densities of the non-interacting systems from those where the assembly of thiols interact with the $\mathrm{Au}(111)$ surface:

$$
\Delta \rho=\rho_{\text {Au-thiols }}-\rho_{A u}-\rho_{\text {thiols }}
$$

where $\Delta \rho$ is the charge density difference and $\rho_{\text {Au-thiols, }} \rho_{\text {Au }}$ and $\rho_{\text {thiols }}$ are the charge densities of the whole system, the isolated Au surface, and the isolated assembly of thiols (both frozen at their final equilibrium configuration upon adsorption), respectively.

The integration of charge density difference throughout the $z$-axis $\left(\Delta \rho_{z}\right)$ has been calculated according to:

$$
\Delta \rho_{z}=\int \Delta \rho d z
$$

The analysis of this property allows a direct comparison of the amount of charge accumulated or depleted at different atomic positions. Figure 12 shows the charge density difference of tpt on $\mathrm{Au}(111)$ (upper panel) and on $\mathrm{Au}_{\mathrm{ad}} / \mathrm{Au}(111)$ (bottom panel). As it can be noticed, in both cases there exists an electron accumulation on the sulfur atom (blue dot), leading to a negative charge. However, immediately below, there is a depletion of electron density between the thiol and the $\mathrm{Au}$ bonded to it, being for the flat surface within the first layer (first red dot) and in the case of the nanostructured surface, on the gold adatom (green dot). These results suggest a higher electron delocalization in the former case and more discrete/localized states in the latter, which confirms our previous assertions. 

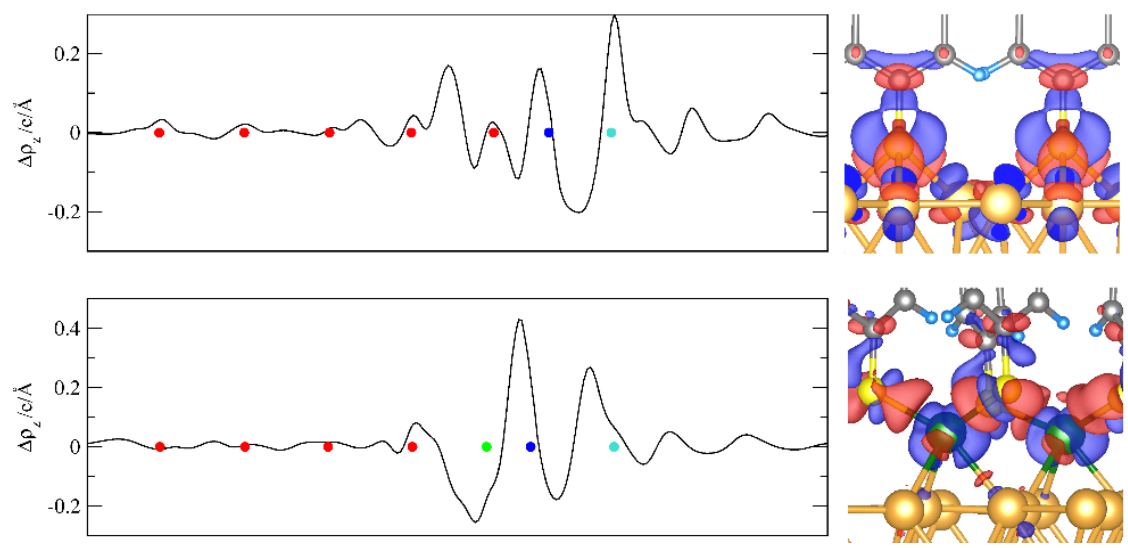

Figure 12. Charge density difference (z-profile and volumetric) for the flat $\mathrm{Au}(111)$ surface (upper panel) and for the nanostructured (bottom panel). The accumulation of charge is in red and the depletion in blue for the volumetric charge density difference.

\section{Conclusions}

In this work the interaction between arylthiols and a Au surface is investigated using periodic DFT. Different SAM organizations were described in literature mainly based on differences in surface reconstructions (flat unreconstructed $\mathrm{Au}(111)$ and adatom $\mathrm{Au}(111)$ surface). DFT energetics on geometrically optimized SAMs and electronic analysis by means of projected density of states (PDOS) and the Crystal Orbital Hamilton Population (COHP) method, was used to interpret the adsorption property differences.

A first conclusion concerning the adsorption geometry underlines the energetically favored $\mathrm{T}$ shaped adsorption compared with horizontal adsorption on unreconstructed $\mathrm{Au}(111)$ surface, for the three studied arylthiols. The long chains were found to adsorb stronger compared with the short chain thiols. However, the adsorption energy difference between the arylthiol chains adsorbed in T-shaped SAMs and adsorbed on flat unreconstructed $\mathrm{Au}(111)$ diminishes markedly from tpt to dpt ( $\Delta=0.39$ ), and remains almost constant between dpt and mpt ( $\Delta=0.23$ and 0.25 ), indicating that the unreconstructed surface becomes more competitive energetically for short chain arylthiols.

Secondly, the nature of the Au-S bond seems to be independent of the chain size. These results are in agreement with the binding energy values. For the thiol- $\mathrm{Au}_{\mathrm{ad}} / \mathrm{Au}(111)$ system, the $3 p$ states of the sulfur directly bonded to the adatom appeared at more positive energies, mainly due to the 
up-shift in the bands of the substrate. Another important feature is that the Au-S interaction is not altered when the aryl chain is substituted by an alkyl chain.

This leads us to conclude that the reconstruction, if any, is only dependent on the side chain interactions. Strong interchain interactions will stabilize surface reconstructions. However, this conclusion is only based on pure thermodynamic considerations. The question that now arises is, if this will be supported by kinetical considerations. This point will of course imply the proposition and the calculation of possible surface reconstruction reaction pathways, which are our next aim. 


\section{Acknowledgements}

This work was performed using HPC resources from GENCI- [CCRT/CINES/IDRIS] (Grant 2016-[x2016082022]) and the CCRE of Université Pierre et Marie Curie. L.F.P. and P.Q. thank the support given by Santa Fe Science Technology and Innovation Agency (ASACTEI, grant 00010-18-2014), CONICET and PICT-2014-1084.

\section{References}

1. Love, J. C.; Estroff, L. A.; Kriebel, J. K.; Nuzzo, R. G.; Whitesides, G. M., SelfAssembled Monolayers of Thiolates on Metals as a Form of Nanotechnology. Chem. Rev. 2005, 105, 1103-1170.

2. Vericat, C.; Vela, M. E.; Benitez, G.; Carro, P.; Salvarezza, R. C., Self-Assembled Monolayers of Thiols and Dithiols on Gold: New Challenges for a Well-Known System. Chem. Soc. Rev. 2010, 39, 1805-1834.

3. Strong, L.; Whitesides, G. M., Structures of Self-Assembled Monolayer Films of Organosulfur Compounds Adsorbed on Gold Single-Crystals - Electron-Diffraction Studies. Langmuir 1988, 4, 546-558.

4. Gottschalck, J.; Hammer, B., A Density Functional Theory Study of the Adsorption of Sulfur, Mercapto, and Methylthiolate on Au(111). J. Chem. Phys. 2002, 116, 784-790.

5. Morikawa, Y.; Liew, C. C.; Nozoye, H., Methylthiolate Induced Vacancy Formation on Au(111): A Density Functional Theoretical Study. Surf. Sci. 2002, 514, 389-393.

6. De Renzi, V.; Di Felice, R.; Marchetto, D.; Biagi, R.; del Pennino, U.; Selloni, A., Ordered (3 X 4) High-Density Phase of Methylthiolate on Au(111). J. Phys. Chem. B 2004, 108, $16-20$

7. Voznyy, O.; Dubowski, J. J.; Yates, J. T., Jr.; Maksymovych, P., The Role of Gold Adatoms and Stereochemistry in Self-Assembly of Methylthiolate on Au(111). J. Am. Chem. Soc. 2009, 131, 12989-12993.

8. Briand, E.; Salmain, M.; Compere, C.; Pradier, C.-M., Immobilization of Protein a on Sams for the Elaboration of Immunosensors. Colloids Surf., B 2006, 53, 215-224.

9. Briand, E.; Salmain, M.; Herry, J.-M.; Perrot, H.; Compere, C.; Pradier, C.-M., Building of an Immunosensor: How Can the Composition and Structure of the Thiol Attachment Layer Affect the Immunosensor Efficiency? Biosens. Bioelectron. 2006, 22, 440-448.

10. Bedford, E.; Humblot, V.; Méthivier, C.; Pradier, C. M.; Gu, F.; Tielens, F.; Boujday, S., An Experimental and Theoretical Approach to Investigate the Effect of Chain Length on Aminothiol Adsorption and Assembly on Gold. Chem. Eur. J. 2015, 21, 14555-14561.

11. Lustemberg, P. G.; Martiarena, M. L.; Martinez, A. E.; Busnengo, H. F., The Reaction Pathways for Hsch3 Adsorption on Au(111): A Density Functional Theory Study. Langmuir 2008, 24, 3274-3279.

12. Hoffling, B.; Ortmann, F.; Hannewald, K.; Bechstedt, F., Adsorption of Cysteine on the Au(110)-Surface: A Density Functional Theory Study; Springer-Verlag Berlin: Berlin, 2010, p 53-60. 
13. Kuhnle, A.; Molina, L. M.; Linderoth, T. R.; Hammer, B.; Besenbacher, F., Growth of Unidirectional Molecular Rows of Cysteine on Au(110)-(1x2) Driven by Adsorbate-Induced Surface Rearrangements. Phys. Rev. Lett. 2004, 93.

14. Di Felice, R.; Selloni, A.; Molinari, E., Dft Study of Cysteine Adsorption on Au(111). $J$. Phys. Chem. B 2003, 107, 1151-1156.

15. Humblot, V.; Tielens, F.; Luque, N. B.; Hampartsoumian, H.; Methivier, C.; Pradier, C. M., Characterization of Two-Dimensional Chiral Self-Assemblies L- and D-Methionine on $\mathrm{Au}(111)$. Langmuir 2014, 30, 203-12.

16. Wang, Y.; Chi, Q.; Zhang, J.; Hush, N. S.; Reimers, J. R.; Ulstrup, J., Chain-Branching Control of the Atomic Structure of Alkanethiol-Based Gold-Sulfur Interfaces. J. Am. Chem. Soc. 2011, 133, 14856-14859.

17. Xu, Q.; Ma, H.; Yip, H.; Jen, A. K. Y., Controlled Assembly of Large Pi-Conjugated Aromatic Thiols on $\mathrm{Au}(111)$. Nanotechnology 2008, 19.

18. Doneux, T.; Tielens, F.; Geerlings, P.; Buess-Herman, C., Experimental and Density Functional Theory Study of the Vibrational Properties of 2-Mercaptobenzimidazole in Interaction with Gold. J. Phys. Chem. A 2006, 110, 11346-52.

19. Costa, D.; Pradier, C.-M.; Tielens, F.; Savio, L., Adsorption and Self-Assembly of BioOrganic Molecules at Model Surfaces: A Route Towards Increased Complexity. Surf. Sci. Rep. 2015, 70, 449-553.

20. Dykman, L.; Khlebtsov, N., Gold Nanoparticles in Biomedical Applications: Recent Advances and Perspectives. Chem. Soc. Rev. 2012, 41, 2256-2282.

21. Dreaden, E. C.; Alkilany, A. M.; Huang, X. H.; Murphy, C. J.; El-Sayed, M. A., The Golden Age: Gold Nanoparticles for Biomedicine. Chem. Soc. Rev. 2012, 41, 2740-2779.

22. Montalti, M.; Prodi, L.; Zaccheroni, N.; Baxter, R.; Teobaldi, G.; Zerbetto, F., Kinetics of Place-Exchange Reactions of Thiols on Gold Nanoparticles. Langmuir 2003, 19, 5172-5174.

23. Brust, M.; Walker, M.; Bethell, D.; Schiffrin, D. J.; Whyman, R., Synthesis of ThiolDerivatised Gold Nanoparticles in a Two-Phase Liquid-Liquid System. J. Chem. Soc., Chem. Commun. 1994, 0, 801-802.

24. Hiramatsu, H.; Osterloh, F. E., A Simple Large-Scale Synthesis of Nearly Monodisperse Gold and Silver Nanoparticles with Adjustable Sizes and with Exchangeable Surfactants. Chem. Mater. 2004, 16, 2509-2511.

25. Zheng, N.; Fan, J.; Stucky, G. D., One-Step One-Phase Synthesis of Monodisperse NobleMetallic Nanoparticles and Their Colloidal Crystals. J. Am. Chem. Soc. 2006, 128, 6550-6551.

26. Woodruff, D. P., The Role of Reconstruction in Self-Assembly of Alkylthiolate Monolayers on Coinage Metal Surfaces. Appl. Surf. Sci. 2007, 254, 76-81.

27. Woodruff, D. P., The Interface Structure of N-Alkylthiolate Self-Assembled Monolayers on Coinage Metal Surfaces. PCCP 2008, 10, 7211-7221.

28. Tielens, F.; Humblot, V.; Pradier, C.-M., Exploring the Reactivity of Mixed OmegaFunctionalized Undecanethiol Self-Assembled Monolayers - a Dft Study. Int. J. Quantum Chem 2008, 108, 1792-1795.

29. Tielens, F.; Humblot, V.; Pradier, C. M.; Calatayud, M.; Illas, F., Stability of Binary Sams Formed by Omega-Acid and Alcohol Functionalized Thiol Mixtures. Langmuir 2009, 25, 99809985.

30. Kresse, G.; Hafner, J., Ab Initio Molecular Dynamics for Liquid Metals. Phys. Rev. B: Condens. Matter 1993, 47, 558-561. 
31. Kresse, G.; Furthmuller, J., Efficient Iterative Schemes for Ab Initio Total-Energy Calculations Using a Plane-Wave Basis Set. Phys. Rev. B: Condens. Matter 1996, 54, 1116911186.

32. Kresse, G.; Furthmuller, J., Efficiency of Ab-Initio Total Energy Calculations for Metals and Semiconductors Using a Plane-Wave Basis Set. Comput. Mater. Sci. 1996, 6, 15-50.

33. Kresse, G.; Hafner, J., Ab Initio Molecular-Dynamics Simulation of the Liquid-Metal Amorphous-Semiconductor Transition in Germanium. Phys. Rev. B: Condens. Matter 1994, 49, 14251-14269.

34. Blochl, P. E., Projector Augmented-Wave Method. Phys. Rev. B: Condens. Matter 1994, 50, 17953-17979.

35. Kresse, G.; Hafner, J., Norm-Conserving and Ultrasoft Pseudopotentials for First-Row and Transition Elements. J. Phys.: Condens. Matter 1994, 6, 8245.

36. Vanderbilt, D., Soft Self-Consistent Pseudopotentials in a Generalized Eigenvalue Formalism. Phys. Rev. B: Condens. Matter 1990, 41, 7892-7895.

37. Kresse, G.; Joubert, D., From Ultrasoft Pseudopotentials to the Projector AugmentedWave Method. Phys. Rev. B: Condens. Matter 1999, 59, 1758-1775.

38. Perdew, J. P.; Burke, K.; Ernzerhof, M., Generalized Gradient Approximation Made Simple [Phys. Rev. Lett. 77, 3865 (1996)]. Phys. Rev. Lett. 1997, 78, 1396-1396.

39. Perdew, J. P.; Burke, K.; Ernzerhof, M., Generalized Gradient Approximation Made Simple. Phys. Rev. Lett. 1996, 77, 3865-3868.

40. Perdew, J. P.; Chevary, J. A.; Vosko, S. H.; Jackson, K. A.; Pederson, M. R.; Singh, D. J.; Fiolhais, C., Erratum: Atoms, Molecules, Solids, and Surfaces: Applications of the Generalized Gradient Approximation for Exchange and Correlation. Phys. Rev. B: Condens. Matter 1993, 48, 4978-4978.

41. Perdew, J. P.; Chevary, J. A.; Vosko, S. H.; Jackson, K. A.; Pederson, M. R.; Singh, D. J.; Fiolhais, C., Atoms, Molecules, Solids, and Surfaces: Applications of the Generalized Gradient Approximation for Exchange and Correlation. Phys. Rev. B: Condens. Matter 1992, 46, 66716687.

42. Perdew, J. P.; Zunger, A., Self-Interaction Correction to Density-Functional Approximations for Many-Electron Systems. Phys. Rev. B: Condens. Matter 1981, 23, 50485079.

43. Kittel, C., Introduction to Solid State Physics, 8th ed. ed.; John Wiley \& sons, Inc.: United States of America, 2005.

44. Monkhorst, H. J.; Pack, J. D., Special Points for Brillouin-Zone Integrations. Phys. Rev. B: Condens. Matter 1976, 13, 5188-5192.

45. Blöchl, P. E.; Jepsen, O.; Andersen, O. K., Improved Tetrahedron Method for BrillouinZone Integrations. Phys. Rev. B: Condens. Matter 1994, 49, 16223-16233.

46. Grimme, S.; Antony, J.; Ehrlich, S.; Krieg, H., A Consistent and Accurate Ab Initio Parametrization of Density Functional Dispersion Correction (Dft-D) for the 94 Elements H-Pu. J. Chem. Phys. 2010, 132, 154104.

47. Grimme, S., Semiempirical Gga-Type Density Functional Constructed with a Long-Range Dispersion Correction. Journal of Computational Chemistry 2006, 27, 1787-1799.

48. Bashir, A.; Azzam, W.; Rohwerder, M.; Terfort, A., Polymorphism in Self-Assembled Terphenylthiolate Monolayers on Au(111). Langmuir 2013, 29, 13449-13456.

49. Wu, X.; Vargas, M. C.; Nayak, S.; Lotrich, V.; Scoles, G., Towards Extending the Applicability of Density Functional Theory to Weakly Bound Systems. J. Chem. Phys. 2001, $115,8748-8757$. 
50. Tielens, F.; Costa, D.; Humblot, V.; Pradier, C. M., Characterization of $\Omega$-Functionalized Undecanethiol Mixed Self-Assembled Monolayers on Au(111): A Combined Polarization Modulation Infrared Reflection-Absorption Spectroscopy/X-Ray Photoelectron Spectroscopy/Periodic Density Functional Theory Study. J. Phys. Chem. C 2008, 112, 182-190.

51. Tielens, F.; Humblot, V.; Pradier, C. M., Exploring the Reactivity of Mixed $\Omega$ Functionalized Undecanethiol Self-Assembled Monolayers - a Dft Study. Int. J. Quantum Chem 2008, 108, 1792-1795.

52. Tielens, F.; Humblot, V.; Pradier, C. M.; Calatayud, M.; Illas, F., Stability of Binary Sams Formed by $\Omega$-Acid and Alcohol Functionalized Thiol Mixtures. Langmuir 2009, 25, 9980-9985.

53. Tielens, F.; Santos, E., Aus and Sh Bond Formation/Breaking During the Formation of Alkanethiol Sams on Au(111): A Theoretical Study. J. Phys. Chem. C 2010, 114, 9444-9452.

54. Luque, N. B.; Santos, E.; Andres, J.; Tielens, F., Effect of Coverage and Defects on the Adsorption of Propanethiol on $\mathrm{Au}(111)$ Surface: A Theoretical Study. Langmuir 2011, 27, 14514-14521.

55. Lofgren, J.; Gronbeck, H.; Moth-Poulsen, K.; Erhart, P., Understanding the Phase Diagram of Self-Assembled Monolayers of Alkanethiolates on Gold. J. Phys. Chem. C 2016, 120, 12059-12067.

56. Forster-Tonigold, K.; Gross, A., A Systematic Dft Study of Substrate Reconstruction Effects Due to Thiolate and Selenolate Adsorption. Surf. Sci. 2015, 640, 18-24.

57. Boto, R. A.; Contreras-García, J.; Calatayud, M., The Role of Dispersion Forces in MetalSupported Self-Assembled Monolayers. Comput. Theor. Chem. 2015, 1053, 322-327.

58. Grimme, S., Do Special Noncovalent П-П Stacking Interactions Really Exist? Angew. Chem. Int. Ed. 2008, 47, 3430-3434.

59. Alonso, M.; Woller, T.; Martín-Martínez, F. J.; Contreras-García, J.; Geerlings, P.; De Proft, F., Understanding the Fundamental Role of $\Pi / \Pi, \Sigma / \Sigma$, and $\Sigma / \Pi$ Dispersion Interactions in Shaping Carbon-Based Materials. Chem. Eur. J. 2014, 20, 4931-4941.

60. Wang, Y.; Hush, N. S.; Reimers, J. R., Formation of Gold-Methanethiyl Self-Assembled Monolayers. J. Am. Chem. Soc. 2007, 129, 14532-3.

61. Fan, X.; Fang, X.; Ran, R.; Ming Lau, W., Density Functional Theory Study of the Adsorption of Methanthiol on Au(1 1 1): Role of Gold Adatoms. Physica E 2014, 59, 248-253.

62. Bareman, J. P.; Klein, M. L., Collective Tilt Behavior in Dense, Substrate-Supported Monolayers of Long-Chain Molecules: A Molecular Dynamics Study. J. Phys. Chem. 1990, 94, 5202-5205.

63. Karaborni, S., Molecular Dynamics Simulations of Long-Chain Amphiphilic Molecules in Langmuir Monolayers. Langmuir 1993, 9, 1334-1343.

64. Frey, S.; Stadler, V.; Heister, K.; Eck, W.; Zharnikov, M.; Grunze, M.; Zeysing, B.; Terfort, A., Structure of Thioaromatic Self-Assembled Monolayers on Gold and Silver. Langmuir 2001, 17, 2408-2415.

65. Yang, G.; Liu, G.-y., New Insights for Self-Assembled Monolayers of Organothiols on $\mathrm{Au}(111)$ Revealed by Scanning Tunneling Microscopy. J. Phys. Chem. B 2003, 107, 8746-8759.

66. Maksymovych, P.; Voznyy, O.; Dougherty, D. B.; Sorescu, D. C.; Yates Jr, J. T., Gold Adatom as a Key Structural Component in Self-Assembled Monolayers of Organosulfur Molecules on Au(1 1 1). Prog. Surf. Sci. 2010, 85, 206-240.

67. Grumelli, D.; Maza, F. L.; Kern, K.; Salvarezza, R. C.; Carro, P., Surface Structure and Chemistry of Alkanethiols on Au(100)- $(1 \times 1)$ Substrates. J. Phys. Chem. C 2016, 120, 291-296.

68. Stettner, J. Self Assembled Monolayer Formation of Alkanethiols on Gold: Growth from Solution Versus Physical Vapor Deposition. Graz University of Technology, Graz, 2010. 
69. Dronskowski, R.; Bloechl, P. E., Crystal Orbital Hamilton Populations (Cohp): EnergyResolved Visualization of Chemical Bonding in Solids Based on Density-Functional Calculations. J. Phys. Chem. 1993, 97, 8617-8624.

\section{TOC}

\section{The Competition between Two High Density Assemblies of Poly Phenyl Thiols on Au(111)}

Leonardo F. Peiretti, Paola Quaino, Frederik Tielens
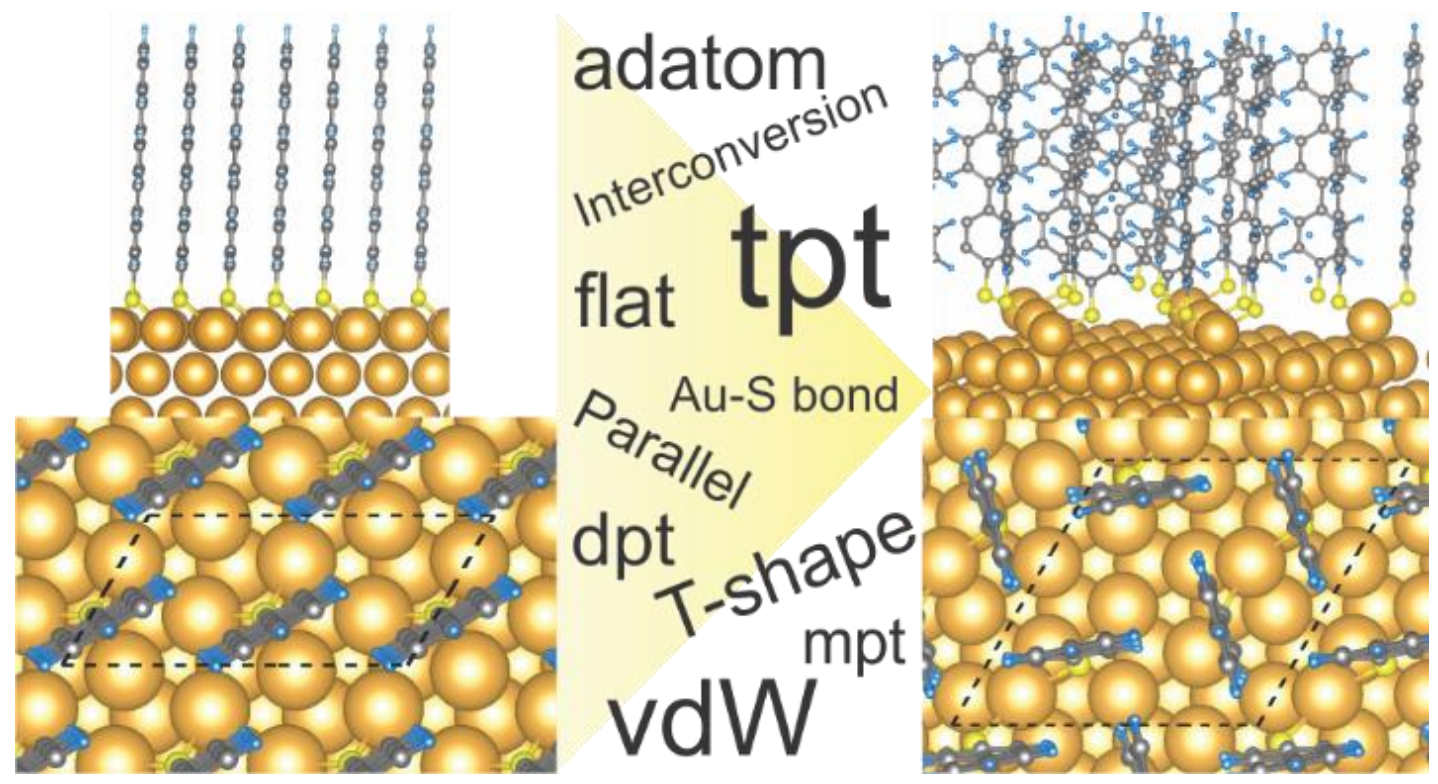